\title{
The Relevance of Dosimetry in Precision Medicine
}

\author{
Michael Lassmann and Uta Eberlein \\ Klinik und Poliklinik für Nuklearmedizin, Universitätsklinikum Würzburg, Würzburg, Germany
}

The aim of this review is to provide an overview of the most recent technologic developments in state-of-the-art equipment and tools for dosimetry in radionuclide therapies. This includes, but is not restricted to, calibration methods for imaging systems. In addition, a summary of new developments that consider the influence of smallscale dosimetry and of biologic effects on radionuclide therapies is given. Finally, the current limitations of patient-specific dosimetry such as bone-marrow dosimetry or dosimetry of a-emitters are discussed.

Key Words: dosimetry; SPECT/CT; calibration; absorbed dose calculation; radiobiology

J Nucl Med 2018; 59:1494-1499

DOI: 10.2967/jnumed.117.206649

\section{A} clear Medicine and Molecular Imaging stated that "in any scientific field concerned with biological effects of radiation, whether for therapy or radiation protection purposes, the effects of radiation on tissue are primarily dependent on the well-established measure absorbed dose" (1). In addition, the basic safety standards directive of the European Union (2) states that radiotherapeutic procedures (which include targeted radionuclide therapies) should be both planned and verified. Consequently, great efforts are needed to assess absorbed doses in cells, tissues, and organs as a prerequisite for treatment planning and for verification of the absorbed doses. These efforts include but are not restricted to quantitative imaging with SPECT or PET, with or without CT (3). A comprehensive overview on the use of theranostic imaging for therapy planning for radioiodine therapy of differentiated thyroid cancer and peptide receptor radionuclide therapy (PRRT) was provided in a recent review article by Eberlein et al. (4). However, dosimetry and treatment planning in radionuclide therapies should surpass simple assessment of absorbed doses and also take into account major biologic effects such as DNA damage and repair mechanisms or the influence of the number and frequency of treatment cycles or of the pharmaceutical itself on biokinetics in normal tissues and tumors. Treatment planning in nuclear medicine should always consider-if technically possible-the safety and the efficacy of a particular treatment.

Received Apr. 20, 2018; revision accepted Jun. 29, 2018.

For correspondence or reprints contact: Michael Lassmann, Klinik und Poliklinik für Nuklearmedizin, Universitätsklinikum Würzburg, Oberdürrbacher Strasse 6, D-97080 Würzburg, Germany.

E-mail: lassmann_m@ukw.de

Published online Jul. 12, 2018.

COPYRIGHT (C) 2018 by the Society of Nuclear Medicine and Molecular Imaging.
Consequently, the aim of this review is to provide an overview of the most recent developments in mathematical and physical methods to increase the accuracy of activity quantifications and absorbed dose calculations in radionuclide therapies. In addition, a summary of new developments that consider the influence of biologic effects on targeted radionuclide therapies will be given. It is not the aim of this review to familiarize the reader with a stepwise approach on how to perform dosimetry.

\section{BASIC PRINCIPLES OF DOSIMETRY IN RADIONUCLIDE THERAPY}

Patient-specific treatment planning and dosimetry include but are not restricted to the following steps:

- the individual (quantitative) measurement of biokinetics in the patient;

- integration of the respective time-activity curves;

- subsequent calculation of the expected absorbed dose relying on the physical properties of the radionuclide and the measured biokinetics (5-8);

- inclusion of model-based predictions of toxicity, for example, for the kidneys (9);

- patient-specific prediction of the activities at which absorbed dose limits will be reached for organs at risk, denoting the onset of deterministic biologic effects such as early or late kidney damage or bone marrow toxicity $(5,10,11)$;

- and, if technically feasible, determination of whether the tumor absorbed doses are sufficient to induce a significant therapeutic effect.

A mandatory prerequisite for quantitative imaging is a traceable calibration of the respective imaging system, including strategies for overcoming partial-volume effects in small structures. Overall, patient-specific dosimetry and treatment planning of radiolabeled substances are essential both for the safety of a treatment and for establishing absorbed dose-response relationships $(5,12,13)$.

\section{THE EVIDENCE BASE FOR INTERNAL DOSIMETRY}

There is sufficient and constantly increasing evidence that treatment outcome correlates with the absorbed doses delivered to tumors and to healthy organs. The findings up to 2014 are summarized in a review article by Strigari et al. for several treatments involving radionuclides, such as radioiodine therapy of benign and malignant thyroid diseases or PRRT of neuroendocrine tumors (13). In recent years, more publications appeared that provided additional evidence for a dose-response relationship in patient treatments. To name a few examples, for the treatment of differentiated thyroid cancer, there are 3 recent reports describing treatment-related dose-effect relationships based on pretherapeutic ${ }^{124}$ I imaging (14-16). For radioimmunotherapies with a novel ${ }^{177}$ Lu-labeled compound addressing the CD-37 antigen, Blakkisrud 
et al. succeeded in establishing a correlation between the hematologic toxicity and absorbed dose to the bone marrow (17).

Overall, the evidence that dosimetry results correlate with patient safety or efficacy is growing constantly. This fact is linked to improvements in technologies for image quantification in nuclear medicine.

\section{NEW DEVELOPMENTS IN MATHEMATICAL AND PHYSICAL METHODS FOR DOSIMETRY}

\section{Image Quantification}

Quantitative imaging is a major part of today's dosimetry and treatment planning workflow. Tomographic methods permit the determination of the activity distribution in a patient at given time points.

PET/CT systems have been used for treatment planning of radionuclide therapies mainly with the radionuclide ${ }^{124} \mathrm{I}(14-16)$. Quantification using this radionuclide is, however, hampered by the presence of a prompt $\gamma$-emission that could potentially lead to false coincidences. Some manufacturers have a built-in prompt $\gamma$-correction, which, for ${ }^{124}$ I, leads to acceptable image quantification (18). Other nuclides that could potentially be used for pretherapeutic PET-based dose planning are ${ }^{44} \mathrm{Sc},{ }^{68} \mathrm{Ga}$, and ${ }^{86} \mathrm{Y}(19)$.

For posttherapeutic dosimetry, imaging of ${ }^{90} \mathrm{Y}$ is proposed after selective internal radiation therapy. The best results are achieved by using systems that include time-of-flight image reconstructions (20).

Modern SPECT/CT systems offer the opportunity for generating patient-specific attenuation maps through CT imaging. Attenuation and scatter corrections can be performed on the projection images, on the reconstructed images, or as part of an iterative reconstruction method (3).

Detailed radionuclide-specific recommendations for the use of SPECT and SPECT/CT systems in general, and in particular for ${ }^{131} \mathrm{I}$ and ${ }^{177} \mathrm{Lu}$, are provided by MIRD pamphlets 23,24 , and 26 (21-23). Recommendations for the setup of PET/CT systems for ${ }^{90} \mathrm{Y}$ PET used for selective internal radiation treatment of liver disease are provided by Willowson et al. (20).

Despite these efforts to provide the users with a comprehensive overview on how to perform quantitative imaging for dosimetry, some questions still need to be addressed. One of them is how best to perform SPECT/CT calibration and quantification, as there is still no general recommendation.

An overview of the most recently applied methods for SPECT/ CT quantification with therapeutically used radionuclides, different phantom geometries, volumes, camera vendors, reconstruction methods, and the related accuracies has been provided by TranGia et al. (24). Table 1 shows only publications with reported accuracies of less than $10 \%(25-32)$.

\section{NOTEWORTHY}

- Improvements in quantification of SPECT/CT images reduce uncertainties in absorbed dose calculations.

- There are still limitations concerning bone marrow dosimetry and dosimetry for therapies with $\alpha$-emitters.

- Pharmacokinetic modeling is about to become an important tool for predicting radiopharmaceutical-related effects.

- For radionuclide therapies, the role of radiobiology in conjunction with dosimetry at a cellular, microscopic, or macroscopic scale needs to be strengthened.
In an international multicenter calibration and standardization trial, Zimmerman et al. (25) found by using calibrated ${ }^{133} \mathrm{Ba}$ sources that SPECT/CT systems showed better reproducibility and better accuracy than planar imaging. The results for SPECT/CT were almost operator-independent.

Wevrett et al. (33) reported on an intercomparison of quantitative imaging of ${ }^{177} \mathrm{Lu}$ in European hospitals using a simple geometry (a shell sphere consisting of 2 isolated concentric spheres allowing the creation of a core filled with a high activity concentration surrounded by a less active background shell). In that study, corrections for partial-volume effects, dead time, or background concentration were not fully incorporated. The authors found an uncertainty of about $20 \%$ for the inner sphere and about $83 \%$ for the outer section. They concluded that the sources of uncertainty should be further researched to fully determine a realistic uncertainty budget.

Siemens Healthineers recently introduced the use of a NISTtraceable calibration source $\left({ }^{75} \mathrm{Se}\right)$ with a $3 \%$ uncertainty $(99 \%$ confidence level) for radionuclides emitting photons with energies between 150 and $250 \mathrm{keV}\left({ }^{123} \mathrm{I},{ }^{111} \mathrm{In},{ }^{177} \mathrm{Lu}\right)$ to ensure standardization of quantitative SPECT/CT (https://www.healthcare.siemens. de/molecular-imaging/xspect/xspect-technology/features\#). Using this calibration method, Tran-Gia et al. (26) showed that a quantification accuracy of less than $2 \%$ could be achieved.

Further efforts at standardizing SPECT/CT calibration are presently being undertaken in the joint European project MRT Dosimetry (http://mrtdosimetry-empir.eu/). Results are expected to be made available by the end of 2019 .

The results of these reports emphasize the need to define a standardized and reproducible calibration across sites for SPECT/ CT quantitative imaging as a prerequisite for dosimetry in multicenter trials.

\section{Volume-of-Interest (VOI) Delineation}

Once the imaging system is calibrated, VOIs need to be drawn to quantify the activity in larger objects. Despite efforts in recent years to provide reproducible operator-independent VOI drawing methods (34-36), a gold standard has yet to be established. Many centers today still rely on operator-dependent manual VOI drawing for image quantification.

Developing a reliable and reproducible method for VOI drawing is also of interest for correcting for partial-volume effects. Presently one method for compensation is, for example, enlarging the VOI around small objects beyond the boundaries of the object, as this might partially account for count losses due to spill-out caused by the limited spatial resolution of the SPECT/CT systems. Another method for partial-volume correction is to derive correction factors by quantifying the activity in CT-based VOIs of spheric objects of varying sizes and to derive correction factors based on the activity in a large object. This method works well for spheres; however, nonspheric objects such as the kidneys might need different partial-volume-error correction factors as has been shown by Robinson et al. (37) and Tran-Gia et al. (38).

\section{Determining Optimal Time Points}

Because dosimetry and treatment planning require quantitative imaging at more than only one time point, the question of the optimal time points for scanning patients still needs to be addressed. General recommendations are provided in MIRD pamphlet 16 (39).

Two recent publications suggested reducing the number of scans needed for dosimetry. Maaß et al. (40) looked into whether the accuracy of treatment planning in PRRT is dependent on the 
TABLE 1

Calibration Methods and Accuracy for Quantitative SPECT Radionuclides Emitting Photons with Energies of More Than $200 \mathrm{keV}$

\begin{tabular}{|c|c|c|c|c|c|c|}
\hline Author & Radionuclide & $\begin{array}{l}\text { Phantom } \\
\text { geometry }\end{array}$ & Volume & System & Reconstruction & Reported accuracy \\
\hline Tran-Gia et al. (26) & ${ }^{177} \mathrm{Lu}$ & Cylinder & $6.8 \mathrm{~L}$ & Siemens & Manufacturer & $1.2 \%$ \\
\hline Beauregard et al. (27) & ${ }^{177} \mathrm{Lu}$ & Cylinder & $1.75-2.5 \mathrm{~L}$ & Siemens & In-house & $\sim 5.6 \%$ \\
\hline D’Arienzo et al. (28) & ${ }^{177} \mathrm{Lu}$ & Cylinder & $4.2 \mathrm{~L}$ & Philips & Manufacturer & $\begin{array}{l}3.7 \% \text { and }-11.6 \% \\
(2 \text { systems })\end{array}$ \\
\hline de Nijs et al. (29) & ${ }^{177} \mathrm{Lu}$ & Sphere & $26.5 \mathrm{~mL}$ & Philips & Manufacturer & $\sim 6.6 \%$ \\
\hline Uribe et al. (30) & ${ }^{177} \mathrm{Lu}$ & Spheres/bottles & $113-199 \mathrm{~mL}$ & Siemens & In-house & $<5 \%$ (objects $>100 \mathrm{~mL}$ ) \\
\hline van Gils et al. (31) & 131 & Large thorax & & Siemens & In-house & $1 \%$ \\
\hline Koral et al. (32) & 131 & Sphere & 7 and $135 \mathrm{~mL}$ & Marconi & In-house & $\begin{array}{l}1 \%-24 \% \\
\quad \text { (volume-dependent) }\end{array}$ \\
\hline Zimmermann et al. (25) & ${ }^{133} \mathrm{Ba}$ & 4 cylinders & $2-23 \mathrm{~mL}$ & Siemens/GE & Manufacturer & $\sim 10 \%$ \\
\hline
\end{tabular}

sampling schedule. When using a priori information from a physiologically based pharmacokinetic model combined with Bayesian information about physiologically based pharmacokinetic model parameter distribution, the administered activity could be determined with acceptable accuracy using only 2 time points $(4 \mathrm{~h}$ and $2 \mathrm{~d}$ ) and thus allow a considerable reduction of needed data for individual dosimetry. Hänscheid et al. (41) suggested that a single quantitative 3-dimensional image might be sufficient to provide values for absorbed doses for PRRT with an accuracy of $10 \%-$ $15 \%$. However, the results of both studies need to be confirmed in larger patient cohorts.

\section{Integration of the Time-Activity Curve and Dose Calculations}

Once the quantitative data have been obtained, the time-activity curves need to be integrated to obtain the time-integrated activity coefficients. The influence of temporal sampling on the results of this integration process has been studied by Guerriero et al. (42). The authors concluded that data should be collected until $100 \mathrm{~h}$ after injection for ${ }^{177} \mathrm{Lu}$ therapies and $70 \mathrm{~h}$ for ${ }^{90} \mathrm{Y}$ therapies. If data collection is stopped earlier, the extrapolation to infinity becomes less accurate, thus influencing the calculation of the time-integrated activity coefficient strongly. Kletting et al. (43) provided a software solution for integrating time-activity curves that includes error propagation and criteria for choosing the optimal fit functions by providing several additional parameters and criteria for the selection.

For the actual dosimetry calculation in radionuclide therapies, appropriate absorbed dose rates per unit activity values ( $\mathrm{S}$ values) either on an organ scale or voxel-based are applied. Organ-based S values provide mean absorbed doses to whole organs or tissues. The most accurate $\mathrm{S}$ values are, at present, provided by Monte Carlo calculations. However, only one publication benchmarked the differences in 3-dimensional dose distributions due to the calculation method of voxel-based $S$ values, showing that the major uncertainty for 3-dimensional dosimetry on clinical SPECT or SPECT/CT images is caused by image blurring and not by differences among the voxel S value calculation methods (44).
Gustafsson et al. (45) established a computer model assessing dosimetry-related uncertainties such as $\gamma$-camera calibration, selection of imaging time points, generation of mass-density maps from CT, SPECT image reconstruction, VOI delineation, partial-volume effects, calculation of absorbed-dose rates, curve fitting, and integration to obtain absorbed dose and biological effective dose (BED). As a result, the authors estimated the importance of different sources of uncertainty. They concluded that the compensation for partial-volume effects via a recovery coefficient and the $\gamma$ camera calibration have the largest impact on the uncertainties.

Ideally, commercially available treatment planning systems for radionuclide therapies should cover all the aforementioned aspects. Presently, commercial software solutions for dosimetry are marketed by Hermes (Hermes Hybrid Dosimetry), Mirada (Simplicit ${ }^{90} \mathrm{Y}$ ), Philips Medical Systems (Stratos), PLANET Dose (DOSIsoft), ABX-CRO Advanced Pharmaceutical Services (QDOSE), and GE Healthcare (Dosimetrix). Only a few of these systems include image reconstruction software for quantitative SPECT or SPECT/CT. Most of the codes rely on adequately quantified images. Some of the companies already obtained a CE (Conformité Européene, or European Conformity) marketing authorization for their software for the purpose of selective internal radiation therapy (Hermes, Mirada, and DOSIsoft).

Because there are no benchmarking tests available for dosimetry software used in radionuclide therapies, efforts are undertaken to produce reference dosimetry data with Monte Carlo simulation software (46). At present, these tests are used in only a few specialized centers. Consequently, the currently available commercial software solutions used for treatment planning need to be carefully evaluated as to whether they suit the respective purpose.

Finally yet importantly, the question remains how best to report the results of a dosimetry study for treatment planning. The European Association of Nuclear Medicine provided some general recommendations on what should be reported and how it should be reported (8). Because the information needed for dosimetry in radionuclide therapy goes beyond providing dose maps in the same format as for externalbeam therapy, the first efforts undertaken by Kesner et al. were to provide a template for reporting a full set of parameters for absorbed dose calculations (47). 


\section{Biology-Driven Improvements}

Another important aspect that takes the individuality of patients into account is the use of pharmacokinetic modeling for predicting absorbed doses or the influence of coadministered nonradioactive compounds. Kletting et al. $(48,49)$ have successfully initiated this approach and have applied this method to PRRTs and to prostatespecific membrane antigen (PSMA) therapies with ${ }^{177} \mathrm{Lu}$. For targeted radionuclides using $\alpha$-particles, compartment modeling is currently the only method for quantifying the influence of the progeny on absorbed doses (50). However, no data on the prospective application of patient-specific pharmacokinetic models have been published.

To further refine absorbed dose calculations with the purpose of including biologic effects such as cell killing and survival, Dale et al. modified the linear-quadratic model, originally developed for radiation oncology, in such a way that it can be applied to radionuclide therapy (10). For kidney dosimetry, the effect of model assumptions on response and the respective implications for radionuclide therapy are compiled in MIRD pamphlet 20 (9). Gustafsson et al. further extended this specific kidney model to better describe the effect of normal-tissue damage repair $(51,52)$. The only available dose-response curves, which are based on BED calculations and agree with external-beam therapy, have been provided for kidney toxicity after PRRT with ${ }^{90}$ Y-labeled DOTA compounds (9). For other radionuclides and organs, with the exception of selective internal radiation treatment, no BED-related organ dose-response data have been published, most likely because of the low toxicity of most of the treatments today with ${ }^{131} \mathrm{I}$ or ${ }^{177} \mathrm{Lu}$.

A combination of both methods-pharmacokinetic modeling and radiobiology — was applied to ${ }^{177} \mathrm{Lu}$-labeled PSMA peptides in a simulation study (53) suggesting that in patients with large, PSMA-positive tumor volumes, higher activities and peptide amounts could safely be administered to maximize tumor BEDs without exceeding the tolerable BED for the organs at risk.

Because ionizing radiation causes DNA damage in normal organs and tissue, it is of great interest to study how this damage is being repaired in patients after radionuclide therapies and, in particular, the relationship to the absorbed doses in normal organs and tissues. Several publications combined patient-specific dosimetry with a quantification of the DNA damage associated with radionuclide therapies (54-57). The authors of these studies showed that the DNA damage in leukocytes after radioiodine therapy for differentiated thyroid cancer and PRRT was associated with the absorbed dose only in the first hours after therapy and was effectively repaired a few days after administration of the radiopharmaceutical. Presently, efforts are under way to extend this method to targeted $\alpha$-therapies (58).

\section{Current Limitations}

The clinical implementation of patient-specific dosimetry currently faces several challenges, despite the technical advances and the increase in knowledge about radiobiology and radiation biology.

Methods for bone marrow dosimetry, although compiled in a comprehensive guideline by the dosimetry committee of the European Association of Nuclear Medicine (7), are still not mature enough to provide robust estimates for absorbed doses to the bone marrow. There have been some promising efforts to correlate the absorbed dose to the bone marrow by imaging methods; however, most have been restricted to a few patients in clinical trials $(13,17,59)$. Further efforts to include MRI in this process have been undertaken but have not yet been introduced to the clinic $(60,61)$.
The gap between organ and tissue dosimetry might be closed by applying small-scale dosimetry models combined with clinical patient biokinetics and compartment modeling. This closure could potentially serve as a bridge between organ and tissue dosimetry and in the interpretation of intrinsic geometric variation and its uncertainties in absorbed dose. An example of this approach in a pretherapy clinical study with ${ }^{111}$ In-ibritumomab tiuxetan was given by Meerkhan et al. (62). The authors focused on the dosimetry for the testicle and presented significant differences in the absorbed dose to the radiosensitive germ cells depending on the location of the radioactive source region and geometry variations of the seminiferous tubule (62). Further research is needed in this area for establishing dose-response relationships.

Dosimetry of $\alpha$-particle emitters for radionuclide therapy based on quantitative imaging still remains a challenge because of the much lower activities that are being administered in comparison to therapies with $\beta$-emitters. Efforts have been undertaken to quantify images for dosimetric purposes after therapies with ${ }^{223} \mathrm{Ra}$-dichloride; however, the errors in quantitative imaging still remain substantial (63-65). To further promote dosimetry in treatments with $\alpha$-particles, methods need to be developed that combine imaging and other patient-specific information such as activity in blood samples as input for tailored pharmacokinetic modeling.

Clinical practice will soon see the introduction of new therapeutic radiopharmaceuticals such as ${ }^{225} \mathrm{Ac}$ - or ${ }^{177} \mathrm{Lu}$-labeled PSMA compounds (66-68), ${ }^{90} \mathrm{Y}$ - or ${ }^{177} \mathrm{Lu}$-labeled chemokine receptor 4 antagonists (69), ${ }^{177} \mathrm{Lu}$-labeled somatostatin antagonists (70), and ${ }^{177} \mathrm{Lu}$-labeled anti-CD37 radioimmunoconjugates (71). In contrast to radioiodine therapy of differentiated thyroid cancer, for example, it is highly likely that most of these therapies will need patient-specific tailoring of the administered activity such that the absorbed dose limits for normal organs and tissues are considered while achieving high absorbed doses to the treatment target.

\section{CONCLUSION}

Prescriptions in radionuclide therapy have been based mostly on a fixed amount of activity for all patients. It is unfortunate that there still have been no randomized controlled trials to evaluate the respective benefits of dosimetry-based versus fixed-activity approaches. However, in the era of precision medicine, the absence of evidence from clinical trials should not hinder the development of dosimetry-based prescriptions and certainly does not justify the absence of posttreatment verification of the absorbed doses delivered, as this verification provides patient-specific information without changing patient management.

The role of radiobiology and radiation biology in examining the impact of radioresistance, low and continuous absorbed dose rates, and heterogeneity of uptake at a cellular, microscopic, or macroscopic scale is under investigation. Dosimetry data will help to expand this field if they are compared with outcomes. We believe that this research field should be strengthened to fully develop the theranostic advantage of radionuclide therapy.

Overall, dosimetry in the era of precision medicine needs fruitful collaborations between different medical specialties in nuclear medicine, oncology, and medical physics. If we succeed in establishing these collaborations, we can foresee a bright future for radionuclide therapy. 


\section{DISCLOSURE}

No potential conflict of interest relevant to this article was reported.

\section{REFERENCES}

1. Flux GD, Sjogreen Gleisner K, Chiesa C, et al. From fixed activities to personalized treatments in radionuclide therapy: lost in translation? Eur J Nucl Med Mol Imaging. 2018;45:152-154.

2. Council of the European Union. European Council Directive 2013/59/Euratom on basic safety standards for protection against the dangers arising from exposure to ionising radiation and repealing Directives 89/618/Euratom, 90/641/Euratom, 96/29/Euratom, 97/43/Euratom and 2003/122/Euratom. Official J Eur Union. 2014; L13:1-73.

3. Flux G, Bardies M, Monsieurs M, Savolainen S, Strands SE, Lassmann M. The impact of PET and SPECT on dosimetry for targeted radionuclide therapy. Z Med Phys. 2006;16:47-59.

4. Eberlein U, Cremonesi M, Lassmann M. Individualized dosimetry for theranostics: necessary, nice to have, or counterproductive? J Nucl Med. 2017(suppl);58: 97S-103S.

5. Sgouros G. Dosimetry of internal emitters. J Nucl Med. 2005;46(suppl):18S-27S.

6. Bolch WE, Eckerman KF, Sgouros G, Thomas SR. MIRD pamphlet no. 21: a generalized schema for radiopharmaceutical dosimetry—standardization of nomenclature. J Nucl Med. 2009;50:477-484.

7. Hindorf C, Glatting G, Chiesa C, Lindén O, Flux G. EANM Dosimetry Committee guidelines for bone marrow and whole-body dosimetry. Eur J Nucl Med Mol Imaging. 2010;37:1238-1250.

8. Lassmann M, Chiesa C, Flux G, Bardies M; EANM Dosimetry Committee. EANM Dosimetry Committee guidance document: good practice of clinical dosimetry reporting. Eur J Nucl Med Mol Imaging. 2011;38:192-200.

9. Wessels BW, Konijnenberg MW, Dale RG, et al. MIRD pamphlet no. 20: the effect of model assumptions on kidney dosimetry and response-implications for radionuclide therapy. $J$ Nucl Med. 2008;49:1884-1899.

10. Dale R, Carabe-Fernandez A. The radiobiology of conventional radiotherapy and its application to radionuclide therapy. Cancer Biother Radiopharm. 2005;20:47-51.

11. Kassis AI. Therapeutic radionuclides: biophysical and radiobiologic principles. Semin Nucl Med. 2008;38:358-366.

12. Strand SE, Jonsson BA, Ljungberg M, Tennvall J. Radioimmunotherapy dosimetry: a review. Acta Oncol. 1993;32:807-817.

13. Strigari L, Konijnenberg M, Chiesa C, et al. The evidence base for the use of internal dosimetry in the clinical practice of molecular radiotherapy. Eur J Nucl Med Mol Imaging. 2014;41:1976-1988.

14. Jentzen W, Hoppenbrouwers J, van Leeuwen P, et al. Assessment of lesion response in the initial radioiodine treatment of differentiated thyroid cancer using ${ }^{124}$ I PET imaging. J Nucl Med. 2014;55:1759-1765.

15. Jentzen W, Verschure F, van Zon A, et al. ${ }^{124}$ I PET assessment of response of bone metastases to initial radioiodine treatment of differentiated thyroid cancer. J Nucl Med. 2016;57:1499-1504.

16. Wierts R, Brans B, Havekes B, et al. Dose-response relationship in differentiated thyroid cancer patients undergoing radioiodine treatment assessed by means of ${ }^{124}$ I PET/CT. J Nucl Med. 2016;57:1027-1032.

17. Blakkisrud J, Londalen A, Dahle J, et al. Red marrow-absorbed dose for nonHodgkin lymphoma patients treated with ${ }^{177} \mathrm{Lu}$-lilotomab satetraxetan, a novel anti-CD37 antibody-radionuclide conjugate. J Nucl Med. 2017;58:55-61.

18. Preylowski V, Schlogl S, Schoenahl F, et al. Is the image quality of I-124-PET impaired by an automatic correction of prompt gammas? PLoS One. 2013;8: e71729.

19. Hetkamp P, Stebner V, Binse I, Jentzen W. Quantitative PET imaging for dosimetry as exemplified by ${ }^{124} \mathrm{I},{ }^{86} \mathrm{Y},{ }^{68} \mathrm{Ga}$, and ${ }^{44} \mathrm{Sc}$. Der Nuklearmediziner. 2018;41:37-51.

20. Willowson KP, Tapner M, Team QI, Bailey DL. A multicentre comparison of quantitative ${ }^{90} \mathrm{Y}$ PET/CT for dosimetric purposes after radioembolization with resin microspheres: the QUEST Phantom Study. Eur J Nucl Med Mol Imaging. 2015;42:1202-1222.

21. Dewaraja YK, Frey EC, Sgouros G, et al. MIRD pamphlet no. 23: quantitative SPECT for patient-specific 3-dimensional dosimetry in internal radionuclide therapy. J Nucl Med. 2012;53:1310-1325.

22. Dewaraja YK, Ljungberg M, Green AJ, et al. MIRD pamphlet no. 24: guidelines for quantitative ${ }^{131}$ I SPECT in dosimetry applications. J Nucl Med. 2013;54: 2182-2188.

23. Ljungberg M, Celler A, Konijnenberg MW, et al. MIRD pamphlet no. 26: joint EANM/MIRD guidelines for quantitative ${ }^{177} \mathrm{Lu}$ SPECT applied for dosimetry of radionuclide therapy. $J$ Nucl Med. 2016;57:151-162.
24. Tran-Gia J, Lassmann M, Kuwert T, Ritt P. Quantitative SPECT/CT imaging for dosimetry. Der Nuklearmediziner. 2018;41:24-36.

25. Zimmerman BE, Grosev D, Buvat I, et al. Multi-centre evaluation of accuracy and reproducibility of planar and SPECT image quantification: an IAEA phantom study. Z Med Phys. 2017;27:98-112.

26. Tran-Gia J, Lassmann M. Characterization of noise and resolution for quantitative ${ }^{177} \mathrm{Lu}$ SPECT/CT with xSPECT quant. $J$ Nucl Med. July 5, 2018 [Epub ahead of print].

27. Beauregard JM, Hofman MS, Pereira JM, Eu P, Hicks RJ. Quantitative ${ }^{177} \mathrm{Lu}$ SPECT (QSPECT) imaging using a commercially available SPECT/CT system. Cancer Imaging. 2011;11:56-66.

28. D'Arienzo M, Cazzato M, Cozzella ML, et al. Gamma camera calibration and validation for quantitative SPECT imaging with ${ }^{177}$ Lu. Appl Radiat Isot. 2016;112:156-164.

29. de Nijs R, Lagerburg V, Klausen TL, Holm S. Improving quantitative dosimetry in ${ }^{177} \mathrm{Lu}$-DOTATATE SPECT by energy window-based scatter corrections. Nucl Med Commun. 2014;35:522-533.

30. Uribe CF, Esquinas PL, Tanguay J, et al. Accuracy of ${ }^{177} \mathrm{Lu}$ activity quantification in SPECT imaging: a phantom study. EJNMMI Phys. 2017;4:2

31. van Gils CA, Beijst C, van Rooij R, de Jong HW. Impact of reconstruction parameters on quantitative I-131 SPECT. Phys Med Biol. 2016;61:5166-5182.

32. Koral KF, Yendiki A, Dewaraja YK. Recovery of total I-131 activity within focal volumes using SPECT and 3D OSEM. Phys Med Biol. 2007;52:777-790.

33. Wevrett J, Fenwick A, Scuffham J, et al. Inter-comparison of quantitative imaging of lutetium-177 $\left({ }^{177} \mathrm{Lu}\right)$ in European hospitals. EJNMMI Phys. 2018;5:17.

34. Pacilio M, Basile C, Shcherbinin S, et al. An innovative iterative thresholding algorithm for tumour segmentation and volumetric quantification on SPECT images: Monte Carlo-based methodology and validation. Med Phys. 2011;38:3050-3061.

35. Gustafsson J, Sundlov A, Sjogreen Gleisner K. SPECT image segmentation for estimation of tumour volume and activity concentration in ${ }^{177} \mathrm{Lu}$-DOTATATE radionuclide therapy. EJNMMI Res. 2017;7:18.

36. Besemer AE, Titz B, Grudzinski JJ, et al. Impact of PET and MRI thresholdbased tumor volume segmentation on patient-specific targeted radionuclide therapy dosimetry using CLR1404. Phys Med Biol. 2017;62:6008-6025.

37. Robinson AP, Tipping J, Cullen DM, et al. Organ-specific SPECT activity calibration using 3D printed phantoms for molecular radiotherapy dosimetry. EJNMMI Phys. 2016;3:12.

38. Tran-Gia J, Lassmann M. Optimizing image quantification for ${ }^{177} \mathrm{Lu}$ SPECT/CT based on a 3D printed 2-compartment kidney phantom. J Nucl Med. 2018;59: 616-624.

39. Siegel JA, Thomas SR, Stubbs JB, et al. MIRD pamphlet no. 16: techniques for quantitative radiopharmaceutical biodistribution data acquisition and analysis for use in human radiation dose estimates. J Nucl Med. 1999;40(suppl):37S-61S.

40. Maaß C, Sachs JP, Hardiansyah D, Mottaghy FM, Kletting P, Glatting G. Dependence of treatment planning accuracy in peptide receptor radionuclide therapy on the sampling schedule. EJNMMI Res. 2016;6:30.

41. Hänscheid H, Lapa C, Buck AK, Lassmann M, Werner RA. Dose mapping after endoradiotherapy with ${ }^{177} \mathrm{Lu}$-DOTATATE/DOTATOC by a single measurement after 4 days. $J$ Nucl Med. 2018;59:75-81.

42. Guerriero F, Ferrari ME, Botta F, et al. Kidney dosimetry in ${ }^{177} \mathrm{Lu}$ and ${ }^{90} \mathrm{Y}$ peptide receptor radionuclide therapy: influence of image timing, time-activity integration method, and risk factors. Biomed Res Int. 2013;2013:935351.

43. Kletting $\mathrm{P}$, Schimmel $\mathrm{S}$, Hanscheid $\mathrm{H}$, et al. The NUKDOS software for treatment planning in molecular radiotherapy. Z Med Phys. 2015;25:264-274.

44. Pacilio M, Amato E, Lanconelli N, et al. Differences in 3D dose distributions due to calculation method of voxel S-values and the influence of image blurring in SPECT. Phys Med Biol. 2015;60:1945-1964.

45. Gustafsson J, Brolin G, Cox M, Ljungberg M, Johansson L, Gleisner KS. Uncertainty propagation for SPECT/CT-based renal dosimetry in ${ }^{177} \mathrm{Lu}$ peptide receptor radionuclide therapy. Phys Med Biol. 2015;60:8329-8346.

46. Chauvin M, Borys D, Botta F, et al. OpenDose: a collaborative effort to produce reference dosimetric data with Monte Carlo simulation software [abstract]. Phys Med. 2017;42(suppl 1):32-33.

47. Kesner AL, Poli GL, Beykan S, Lassmann M. The IAEA Radiotracer Biodistribution Template: a community resource for supporting the standardization and reporting of radionuclide pre-dosimetry data. Phys Med. 2017;44:83-85.

48. Kletting $\mathrm{P}$, Schuchardt C, Kulkarni HR, et al. Investigating the effect of ligand amount and injected therapeutic activity: a simulation study for ${ }^{177} \mathrm{Lu}$-labeled PSMA-targeting peptides. PLoS One. 2016;11:e0162303.

49. Kletting P, Kull T, Maass C, et al. Optimized peptide amount and activity for ${ }^{90}$ Y-labeled DOTATATE therapy. J Nucl Med. 2016;57:503-508.

50. Lassmann M, Nosske D. Dosimetry of ${ }^{223}$ Ra-chloride: dose to normal organs and tissues. Eur J Nucl Med Mol Imaging. 2013;40:207-212. 
51. Gustafsson J, Nilsson P, Gleisner KS. On the biologically effective dose (BED)using convolution for calculating the effects of repair: II. Numerical considerations. Phys Med Biol. 2013;58:1529-1548.

52. Gustafsson J, Nilsson P, Gleisner KS. On the biologically effective dose (BED)using convolution for calculating the effects of repair: I. Analytical considerations. Phys Med Biol. 2013;58:1507-1527.

53. Begum NJ, Thieme A, Eberhardt N, et al. The effect of total tumor volume on the biologically effective dose to tumor and kidneys for ${ }^{177} \mathrm{Lu}$-labeled PSMA peptides. J Nucl Med. 2018;59:929-933.

54. Lassmann M, Hanscheid $\mathrm{H}$, Gassen D, et al. In vivo formation of gamma-H2AX and 53BP1 DNA repair foci in blood cells after radioiodine therapy of differentiated thyroid cancer. J Nucl Med. 2010;51:1318-1325.

55. Eberlein U, Nowak C, Bluemel C, et al. DNA damage in blood lymphocytes in patients after ${ }^{177} \mathrm{Lu}$ peptide receptor radionuclide therapy. Eur J Nucl Med Mol Imaging. 2015;42:1739-1749.

56. Denoyer D, Lobachevsky P, Jackson P, Thompson M, Martin OA, Hicks RJ. Analysis of ${ }^{177} \mathrm{Lu}$-DOTA-octreotate therapy-induced DNA damage in peripheral blood lymphocytes of patients with neuroendocrine tumors. J Nucl Med. 2015; 56:505-511.

57. Eberlein U, Scherthan H, Bluemel C, et al. DNA damage in peripheral blood lymphocytes of thyroid cancer patients after radioiodine therapy. J Nucl Med. 2016;57:173-179.

58. Schumann S, Eberlein U, Muhtadi R, Lassmann M, Scherthan H. DNA damage in leukocytes after internal ex-vivo irradiation of blood with the alpha-emitter Ra-223. Sci Rep. 2018;8:2286.

59. Walrand S, Barone R, Pauwels S, Jamar F. Experimental facts supporting a red marrow uptake due to radiometal transchelation in ${ }^{90}$ Y-DOTATOC therapy and relationship to the decrease of platelet counts. Eur J Nucl Med Mol Imaging. 2011;38:1270-1280.

60. Pichardo JC, Milner RJ, Bolch WE. MRI measurement of bone marrow cellularity for radiation dosimetry. J Nucl Med. 2011;52:1482-1489.

61. Salas-Ramirez M, Tran-Gia J, Kesenheimer C, et al. Quantification of fat fraction in lumbar vertebrae: correlation with age and implications for bone marrow dosimetry in molecular radiotherapy. Phys Med Biol. 2018;63:025029.
62. Meerkhan SA, Sjogreen-Gleisner K, Larsson E, Strand SE, Jonsson BA. Testis dosimetry in individual patients by combining a small-scale dosimetry model and pharmacokinetic modeling: application of ${ }^{111} \mathrm{In}$-ibritumomab tiuxetan (Zevalin ${ }^{\circledR}$ ). Phys Med Biol. 2014;59:7889-7904.

63. Hindorf C, Chittenden S, Aksnes AK, Parker C, Flux GD. Quantitative imaging of ${ }^{223}$ Ra-chloride (Alpharadin) for targeted alpha-emitting radionuclide therapy of bone metastases. Nucl Med Commun. 2012;33:726-732.

64. Pacilio M, Ventroni G, De Vincentis G, et al. Dosimetry of bone metastases in targeted radionuclide therapy with alpha-emitting ${ }^{223}$ Ra-dichloride. Eur J Nucl Med Mol Imaging. 2016;43:21-33.

65. Pacilio M, Cassano B, Chiesa C, et al. The Italian multicentre dosimetric study for lesion dosimetry in ${ }^{223} \mathrm{Ra}$ therapy of bone metastases: calibration protocol of gamma cameras and patient eligibility criteria. Phys Med. 2016;32:17311737.

66. Kratochwil C, Bruchertseifer F, Giesel FL, et al. ${ }^{225}$ Ac-PSMA-617 for PSMAtargeted alpha-radiation therapy of metastatic castration-resistant prostate cancer. J Nucl Med. 2016;57:1941-1944.

67. Kratochwil C, Giesel FL, Stefanova M, et al. PSMA-targeted radionuclide therapy of metastatic castration-resistant prostate cancer with ${ }^{177} \mathrm{Lu}$-labeled PSMA617. J Nucl Med. 2016;57:1170-1176.

68. Weineisen M, Schottelius M, Simecek J, et al. ${ }^{68} \mathrm{Ga}$ - and ${ }^{177} \mathrm{Lu}$-labeled PSMA I\&T: optimization of a PSMA-targeted theranostic concept and first proof-ofconcept human studies. J Nucl Med. 2015;56:1169-1176.

69. Herrmann K, Schottelius M, Lapa C, et al. First-in-human experience of CXCR4-directed endoradiotherapy with ${ }^{177} \mathrm{Lu}$ - and ${ }^{90}$ Y-labeled pentixather in advanced-stage multiple myeloma with extensive intra- and extramedullary disease. J Nucl Med. 2016;57:248-251.

70. Wild D, Fani M, Fischer R, et al. Comparison of somatostatin receptor agonist and antagonist for peptide receptor radionuclide therapy: a pilot study. $J$ Nucl Med. 2014;55:1248-1252.

71. Blakkisrud J, Holtedahl JE, Londalen A, et al. Biodistribution and dosimetry results from a phase 1 trial of therapy with the antibody-radionuclide conjugate ${ }^{177}$ Lu-lilotomab satetraxetan. J Nucl Med. 2018;59:704-710. 